Living Rev. Relativity, 8, (2005), 2

http://www.livingreviews.org/lrr-2005-2

(Update of lrr-2002-7)

\title{
The Einstein-Vlasov System/Kinetic Theory
}

\author{
Håkan Andréasson \\ Department of Mathematics \\ Chalmers University of Technology \\ S-412 96 Göteborg, Sweden \\ email: hand@math.chalmers.se \\ http://www.math. chalmers.se/ hand \\ Accepted on 23 February 2005 \\ Published on 18 March 2005 \\ Living Reviews in Relativity \\ Published by the \\ Max Planck Institute for Gravitational Physics \\ (Albert Einstein Institute) \\ Am Mühlenberg 1, 14424 Golm, Germany \\ ISSN 1433-8351
}

\begin{abstract}
The main purpose of this article is to provide a guide to theorems on global properties of solutions to the Einstein-Vlasov system. This system couples Einstein's equations to a kinetic matter model. Kinetic theory has been an important field of research during several decades in which the main focus has been on nonrelativistic and special relativistic physics, i.e. to model the dynamics of neutral gases, plasmas, and Newtonian self-gravitating systems. In 1990, Rendall and Rein initiated a mathematical study of the Einstein-Vlasov system. Since then many theorems on global properties of solutions to this system have been established. The Vlasov equation describes matter phenomenologically, and it should be stressed that most of the theorems presented in this article are not presently known for other such matter models (i.e. fluid models). This paper gives introductions to kinetic theory in non-curved spacetimes and then the Einstein-Vlasov system is introduced. We believe that a good understanding of kinetic theory in non-curved spacetimes is fundamental to good comprehension of kinetic theory in general relativity.
\end{abstract}

(c) Max Planck Society and the authors.

Further information on copyright is given at http://relativity.livingreviews .org/About/copyright.html

For permission to reproduce the article please contact livrev@aei.mpg.de. 


\section{How to cite this article}

Owing to the fact that a Living Reviews article can evolve over time, we recommend to cite the article as follows:

Håkan Andréasson,

"The Einstein-Vlasov System/Kinetic Theory",

Living Rev. Relativity, 8, (2005), 2. [Online Article]: cited [<date $>$ ],

http://www.livingreviews.org/lrr-2005-2

The date given as $<$ date $>$ then uniquely identifies the version of the article you are referring to.

\section{Article Revisions}

Living Reviews supports two different ways to keep its articles up-to-date:

Fast-track revision A fast-track revision provides the author with the opportunity to add short notices of current research results, trends and developments, or important publications to the article. A fast-track revision is refereed by the responsible subject editor. If an article has undergone a fast-track revision, a summary of changes will be listed here.

Major update A major update will include substantial changes and additions and is subject to full external refereeing. It is published with a new publication number.

For detailed documentation of an article's evolution, please refer always to the history document of the article's online version at http: //www.livingreviews.org/lrr-2005-2. 


\section{Contents}

1 Introduction to Kinetic Theory $\quad \mathbf{5}$

1.1 The relativistic Boltzmann equation . . . . . . . . . . . . . . . . . 5

1.2 The Vlasov-Maxwell and Vlasov-Poisson systems . . . . . . . . . . . . . . . . . 8

1.3 The Nordström-Vlasov system . . . . . . . . . . . . . . . . . . . . . 10

1.4 The Einstein-Vlasov system . . . . . . . . . . . . . . . . . . . . 11

2 Global Existence Theorems for the Einstein-Vlasov System 14

2.1 Spherically symmetric spacetimes . . . . . . . . . . . . . . . . 14

2.2 Cosmological solutions . . . . . . . . . . . . . . . . . . . . . . . . . . . . 18

2.3 Cosmological models with a cosmological constant or a scalar field . . . . . . . . 20

3 Stationary Solutions to the Einstein-Vlasov System 23

4 Acknowledgements $r 25$

$\begin{array}{lr}\text { References } & 33\end{array}$ 



\section{Introduction to Kinetic Theory}

In general relativity, kinetic theory has been used relatively sparsely to model phenomenological matter in comparison to fluid models. From a mathematical point of view, however, there are fundamental advantages to using a kinetic description. In non-curved spacetimes kinetic theory has been studied intensively as a mathematical subject during several decades, and it has also played an important role from an engineering point of view. In the first part of this introduction, we will review kinetic theory in non-curved spacetimes and we will consider mainly the special relativistic case, but mathematical results in the nonrelativistic case will also be discussed. We believe that a good understanding of kinetic theory in non-curved spacetimes is fundamental to good comprehension of kinetic theory in general relativity. Moreover, it is often the case that mathematical methods used to treat the Einstein-Vlasov system are carried over from methods developed in the special relativistic or nonrelativistic case.

The purpose of kinetic theory is to model the time evolution of a collection of particles. The particles may be entirely different objects depending on the physical situation. For instance, the particles are atoms and molecules in a neutral gas or electrons and ions in a plasma. In stellar dynamics the particles are stars and in a cosmological case they are galaxies or even clusters of galaxies. Mathematical models of particle systems are most frequently described by kinetic or fluid equations. A characteristic feature of kinetic theory is that its models are statistical and the particle systems are described by distribution functions $f=f(t, x, p)$, which represent the density of particles with given spacetime position $(t, x) \in \mathbb{R} \times \mathbb{R}^{3}$ and momentum $p \in \mathbb{R}^{3}$. A distribution function contains a wealth of information, and macroscopical quantities are easily calculated from this function. In a fluid model the quantities that describe the system do not depend on the momentum $p$ but only on the spacetime point $(t, x)$. A choice of model is usually made with regard to the physical properties of interest for the system or with regard to numerical considerations. It should be mentioned that a fluid model that is too naive may give rise to shellcrossing singularities, which are unphysical. In a kinetic description such phenomena are ruled out.

The time evolution of the system is determined by the interactions between the particles which depend on the physical situation. For instance, the driving mechanism for the time evolution of a neutral gas is the collision between particles (the relativistic Boltzmann equation). For a plasma the interaction is through the electric charges (the Vlasov-Maxwell system), and in the stellar and cosmological cases the interaction is gravitational (the Einstein-Vlasov system). Of course, combinations of interaction processes are also considered but in many situations one of them is strongly dominating and the weaker processes are neglected.

\subsection{The relativistic Boltzmann equation}

Consider a collection of neutral particles in Minkowski spacetime. Let the signature of the metric be $(-,+,+,+)$, let all the particles have rest mass $m=1$, and normalize the speed of light $c$ to one. The four-momentum of a particle is denoted by $p^{a}, a=0,1,2,3$. Since all particles have equal rest mass, the four-momentum for each particle is restricted to the mass shell, $p^{a} p_{a}=-m^{2}=-1$. Thus, by denoting the three-momentum by $p \in \mathbb{R}^{3}, p^{a}$ may be written $p^{a}=\left(-p^{0}, p\right)$, where $|p|$ is the usual Euclidean length of $p$ and $p^{0}=\sqrt{1+|p|^{2}}$ is the energy of a particle with three-momentum $p$. The relativistic velocity of a particle with momentum $p$ is denoted by $\hat{p}$ and is given by

$$
\hat{p}=\frac{p}{\sqrt{1+|p|^{2}}} .
$$


Note that $|\hat{p}|<1=c$. The relativistic Boltzmann equation models the spacetime behaviour of the one-particle distribution function $f=f(t, x, p)$, and it has the form

$$
\left(\partial_{t}+\frac{p}{p^{0}} \cdot \nabla_{x}\right) f=Q(f, f)
$$

where the relativistic collision operator $Q(f, g)$ is defined by

$$
Q(f, g)=\int_{\mathbb{R}^{3}} \int_{\mathbb{S}^{2}} k(p, q, \omega)[f(p+a(p, q, \omega) \omega) g(q-a(p, q, \omega) \omega)-f(p) g(q)] d p d \omega .
$$

(Note that $g=f$ in Equation (2)). Here $d \omega$ is the element of surface area on $\mathbb{S}^{2}$ and $k(p, q, \omega)$ is the scattering kernel, which depends on the scattering cross-section in the interaction process. See [34] for a discussion about the scattering kernel. The function $a(p, q, \omega)$ results from the collision mechanics. If two particles, with momentum $p$ and $q$ respectively, collide elastically (no energy loss) with scattering angle $\omega \in \mathbb{S}^{2}$, their momenta will change, $p \rightarrow p^{\prime}$ and $q \rightarrow q^{\prime}$. The relation between $p, q$ and $p^{\prime}, q^{\prime}$ is

$$
p^{\prime}=p+a(p, q, \omega) \omega, \quad q^{\prime}=q-a(p, q, \omega) \omega,
$$

where

$$
a(p, q, \omega)=\frac{2\left(p^{0}+q^{0}\right) p^{0} q^{0}(\omega \cdot(\hat{q}-\hat{p}))}{\left(p^{0}+q^{0}\right)^{2}-(\omega \cdot(p+q))^{2}} .
$$

This relation is a consequence of four-momentum conservation,

$$
p^{a}+q^{a}=p^{a^{\prime}}+q^{a^{\prime}}
$$

or equivalently

$$
\begin{aligned}
p^{0}+q^{0} & =p^{0^{\prime}}+q^{0^{\prime}}, \\
p+q & =p^{\prime}+q^{\prime} .
\end{aligned}
$$

These are the conservation equations for relativistic particle dynamics. In the classical case these equations read

$$
\begin{aligned}
|p|^{2}+|q|^{2} & =\left|p^{\prime}\right|^{2}+\left|q^{\prime}\right|^{2}, \\
p+q & =p^{\prime}+q^{\prime} .
\end{aligned}
$$

The function $a(p, q, \omega)$ is the distance between $p$ and $p^{\prime}\left(q\right.$ and $q^{\prime}$ ), and the analogue function in the Newtonian case has the form

$$
a_{\mathrm{cl}}(p, q, \omega)=\omega \cdot(q-p) .
$$

By inserting $a_{\mathrm{cl}}$ in place of $a$ in Equation (3) we obtain the classical Boltzmann collision operator (disregarding the scattering kernel, which is also different). In [20] classical solutions to the relativistic Boltzmann equations are studied as $c \rightarrow \infty$, and it is proved that the limit as $c \rightarrow \infty$ of these solutions satisfy the classical Boltzmann equation.

The main result concerning the existence of solutions to the classical Boltzmann equation is a theorem by DiPerna and Lions [36] that proves existence, but not uniqueness, of renormalized solutions (i.e. solutions in a weak sense, which are even more general than distributional solutions). An analogous result holds in the relativistic case, as was shown by Dudyńsky and Ekiel-Jezewska [37]. Regarding classical solutions, Illner and Shinbrot [58] have shown global existence of solutions to the nonrelativistic Boltzmann equation for small initial data (close to vacuum). At present there

Living Reviews in Relativity

http: //www. livingreviews . org//rr-2005-2 
is no analogous result for the relativistic Boltzmann equation and this must be regarded as an interesting open problem. There is however a recent result [74] for the homogeneous relativistic Boltzmann equation where global existence is shown for small initial data and bounded scattering kernel. When the data are close to equilibrium (see below), global existence of classical solutions has been proved by Glassey and Strauss [48] in the relativistic case and by Ukai [115] in the nonrelativistic case (see also [108] and [69]).

The collision operator $Q(f, g)$ may be written in an obvious way as

$$
Q(f, g)=Q^{+}(f, g)-Q^{-}(f, g),
$$

where $Q^{+}$and $Q^{-}$are called the gain and loss term, respectively. If the loss term is deleted the gain-term-only Boltzmann equation is obtained. It is interesting to note that the methods of proof for the small data results mentioned above concentrate on gain-term-only equations, and once that is solved it is easy to include the loss term. In [5] it is shown that the gain-term-only classical and relativistic Boltzmann equations blow up for initial data not restricted to a small neighbourhood of trivial data. Thus, if a global existence proof for unrestricted data will be given it will necessarily use the full collision operator.

The gain term has a nice regularizing property in the momentum variable. In [2] it is proved that given $f \in L^{2}\left(\mathbb{R}^{3}\right)$ and $g \in L^{1}\left(\mathbb{R}^{3}\right)$ with $f, g \geq 0$, then

$$
\left\|Q^{+}(f, g)\right\|_{H^{1}\left(\mathbb{R}_{p}^{3}\right)} \leq C\|f\|_{L^{2}\left(\mathbb{R}_{p}^{3}\right)}\|g\|_{L^{1}\left(\mathbb{R}_{p}^{3}\right)},
$$

under some technical requirements on the scattering kernel. Here $H^{s}$ is the usual Sobolev space. This regularizing result was first proved by P.L. Lions [65] in the classical situation. The proof relies on the theory of Fourier integral operators and on the method of stationary phase, and requires a careful analysis of the collision geometry, which is very different in the relativistic case. See also [119] and [120] for a simplified proof in the classical and relativistic case respectively.

The regularizing theorem has many applications. An important application is to prove that solutions tend to equilibrium for large times. More precisely, Lions used the regularizing theorem to prove that solutions to the (classical) Boltzmann equation, with periodic boundary conditions, converge in $L^{1}$ to a global Maxwellian,

$$
M=e^{-\alpha|p|^{2}+\beta \cdot p+\gamma} \quad \text { with } \alpha, \gamma \in R, \quad \alpha>0, \quad \beta \in \mathbb{R}^{3},
$$

as time goes to infinity. This result had first been obtained by Arkeryd [10] by using non-standard analysis. It should be pointed out that the convergence takes place through a sequence of times tending to infinity and it is not known whether the limit is unique or depends on the sequence. In the relativistic situation, the analogous question of convergence to a relativistic Maxwellian, or a Jüttner equilibrium solution,

$$
J=e^{-\alpha \sqrt{1+|p|^{2}}+\beta \cdot p+\gamma}, \quad \alpha, \beta, \text { and } \gamma \text { as above, with } \alpha>|\beta|,
$$

had been studied by Glassey and Strauss [48, 49]. In the periodic case they proved convergence in a variety of function spaces for initial data close to a Jüttner solution. Having obtained the regularizing theorem for the relativistic gain term, it is a straightforward task to follow the method of Lions and prove convergence to a local Jüttner solution for arbitrary data (satisfying the natural bounds of finite energy and entropy) that is periodic in the space variables. In [2] it is next proved that the local Jüttner solution must be a global one, due to the periodicity of the solution.

For more information on the relativistic Boltzmann equation on Minkowski space we refer to $[41,34,110]$ and in the nonrelativistic case we refer to the excellent review paper by Villani [116] and the books [41, 24]. 


\subsection{The Vlasov-Maxwell and Vlasov-Poisson systems}

Let us consider a collisionless plasma, which is a collection of particles for which collisions are relatively rare and the interaction is through their charges. We assume below that the plasma consists only of one type of particle, whereas the results below also hold for plasmas with several particle species. The particle rest mass is normalized to one. In the kinetic framework, the most general set of equations for modelling a collisionless plasma is the relativistic Vlasov-Maxwell system:

$$
\begin{array}{rlrl}
\partial_{t} f+\hat{v} \cdot \nabla_{x} f+(E(t, x)+\hat{v} \times B(t, x)) \cdot \nabla_{v} f & =0 \\
\partial_{t} E+j & =c \nabla \times B, & \nabla \cdot E & =\rho, \\
\partial_{t} B & =-c \nabla \times E, & \nabla \cdot B & =0 .
\end{array}
$$

The notation follows the one already introduced with the exception that the momenta are now denoted by $v$ instead of $p$. This has become a standard notation in this field. $E$ and $B$ are the electric and magnetic fields, and $\hat{v}$ is the relativistic velocity,

$$
\hat{v}=\frac{v}{\sqrt{1+|v|^{2} / c^{2}}},
$$

where $c$ is the speed of light. The charge density $\rho$ and current $j$ are given by

$$
\rho=\int_{\mathbb{R}^{3}} f d v, \quad j=\int_{\mathbb{R}^{3}} \hat{v} f d v .
$$

Equation (12) is the relativistic Vlasov equation and Equations $(13,14)$ are the Maxwell equations.

A special case in three dimensions is obtained by considering spherically symmetric initial data. For such data it can be shown that the solution will also be spherically symmetric, and that the magnetic field has to be constant. The Maxwell equation $\nabla \times E=-\partial_{t} B$ then implies that the electric field is the gradient of a potential $\phi$. Hence, in the spherically symmetric case the relativistic Vlasov-Maxwell system takes the form

$$
\begin{aligned}
& \partial_{t} f+\hat{v} \cdot \nabla_{x} f+\beta E(t, x) \cdot \nabla_{v} f=0, \\
& E=\nabla \phi, \quad \Delta \phi=\rho .
\end{aligned}
$$

Here $\beta=1$, and the constant magnetic field has been set to zero, since a constant field has no significance in this discussion. This system makes sense for any initial data, without symmetry constraints, and is called the relativistic Vlasov-Poisson equation. Another special case of interest is the classical limit, obtained by letting $c \rightarrow \infty$ in Equations (12, 13, 14), yielding:

$$
\begin{array}{r}
\partial_{t} f+v \cdot \nabla_{x} f+\beta E(t, x) \cdot \nabla_{v} f=0, \\
E=\nabla \phi, \quad \Delta \phi=\rho,
\end{array}
$$

where $\beta=1$. See Schaeffer [105] for a rigorous derivation of this result. This is the (nonrelativistic) Vlasov-Poisson equation, and $\beta=1$ corresponds to repulsive forces (the plasma case). Taking $\beta=-1$ means attractive forces and the Vlasov-Poisson equation is then a model for a Newtonian self-gravitating system.

One of the fundamental problems in kinetic theory is to find out whether or not spontaneous shock formations will develop in a collisionless gas, i.e. whether solutions to any of the equations above will remain smooth for all time, given smooth initial data.

If the initial data are small this problem has an affirmative solution in all cases considered above (see $[42,47,12,13])$. For initial data unrestricted in size the picture is more involved. In

Living Reviews in Relativity

http: //www. livingreviews. org/lrr-2005-2 
order to obtain smooth solutions globally in time, the main issue is to control the support of the momenta

$$
Q(t):=\sup \left\{|v|: \exists(s, x) \in[0, t] \times \mathbb{R}^{3} \text { such that } f(s, x, v) \neq 0\right\},
$$

i.e. to bound $Q(t)$ by a continuous function so that $Q(t)$ will not blow up in finite time. That such a control is sufficient for obtaining global existence of smooth solutions follows from well-known results in the different cases (see [46, 54, 14, 42]). For the full three-dimensional relativistic VlasovMaxwell system, this important problem of establishing whether or not solutions will remain smooth for all time is open. There has been an increased activity during the last years aiming at a solution of this problem. Two new methods of proofs of the same theorem as in [46] are given in $[60,17]$. A different sufficient criterion for global existence is given by Pallard in [77] and he also shows a new bound for the electromagnetic field in terms of $Q(t)$ in [78]. In two space and three momentum dimensions, Glassey and Schaeffer [43] have shown that $Q(t)$ can be controlled, which thus yields global existence of smooth solutions in that case (see also [44]).

The relativistic and nonrelativistic Vlasov-Poisson equations are very similar in form. In particular, the equation for the field is identical in the two cases. However, the mathematical results concerning the two systems are very different. In the nonrelativistic case Batt [14] gave an affirmative solution 1977 in the case of spherically symmetric data. Pfaffelmoser [80] (see also Schaeffer [107]) was the first one to give a proof for general smooth data. He obtained the bound

$$
Q(t) \leq C(1+t)^{(51+\delta) / 11},
$$

where $\delta>0$ could be taken arbitrarily small. This bound was later improved by different authors. The sharpest bound valid for $\beta=1$ and $\beta=-1$ has been given by Horst [55] and reads

$$
Q(t) \leq C(1+t) \log (2+t) .
$$

In the case of repulsive forces $(\beta=1)$ Rein [83] has found the sharpest estimate by using a new identity for the Vlasov-Poisson equation, discovered independently by Illner and Rein [57] and by Perthame [79]. Rein's estimate reads

$$
Q(t) \leq C(1+t)^{2 / 3} .
$$

Independently and about the same time as Pfaffelmoser gave his proof, Lions and Perthame [66] used a different method for proving global existence. To some extent their method seems to be more generally applicable to attack problems similar to the Vlasov-Poisson equation but which are still quite different (see $[3,61]$ ). On the other hand, their method does not give such strong growth estimates on $Q(t)$ as described above. For the relativistic Vlasov-Poisson equation, Glassey and Schaeffer [42] showed in the case $\beta=1$ that if the data are spherically symmetric, $Q(t)$ can be controlled, which is analogous to the result by Batt mentioned above. Also in the case of cylindrical symmetry they are able to control $Q(t)$; see [45]. If $\beta=-1$ it was also shown in [42] that blowup occurs in finite time for spherically symmetric data with negative total energy. This system, however, is unphysical in the sense that it is not a special case of the Einstein-Vlasov system. Quite surprisingly, for general smooth initial data none of the techniques discussed above for the nonrelativistic Vlasov-Poisson equation apply in the relativistic case. This fact is annoying since it has been suggested that an understanding of this equation may be necessary for understanding the three-dimensional relativistic Vlasov-Maxwell equation. However, the relativistic Vlasov-Poisson equation lacks the Lorentz invariance; it is a hybrid of a classical Galilei invariant field equation and a relativistic transport equation (17). Only for spherical symmetric data is the equation a fundamental physical equation. The classical Vlasov-Poisson equation is on the other hand Galilean invariant. In [1] a different equation for the field is introduced that is observer independent among Lorentz observers. By coupling this equation for the field to the relativistic Vlasov equation, 
the function $Q(t)$ may be controlled as shown in [1]. This is an indication that the transformation properties are important in studying existence of smooth solutions (the situation is less subtle for weak solutions, where energy estimates and averaging are the main tools (see [56, 35, 86]). Hence, it is unclear whether or not the relativistic Vlasov-Poisson equation will play a central role in the understanding of the Lorentz invariant relativistic Vlasov-Maxwell equation.

We refer to the book by Glassey [41] for more information on the relativistic Vlasov-Maxwell system and the Vlasov-Poisson equation.

\subsection{The Nordström-Vlasov system}

Before turning to the main theme of this review, i.e. the Einstein-Vlasov system, we introduce a system which has many mathematical features in common with the Vlasov-Maxwell system and which recently has been mathematically studied for the first time. It is based on an alternative theory of gravity which was given by Nordström [70] in 1913. By coupling this model to a kinetic description of matter the Nordström-Vlasov system results. In Nordström gravity the scalar field $\phi$ contains the gravitational effects as described below. The Nordström-Vlasov system reads

$$
\begin{aligned}
& \partial_{t}^{2} \phi-\triangle_{x} \phi=-e^{4 \phi} \int \mathfrak{f} \frac{d p}{\sqrt{1+|p|^{2}}}, \\
& \partial_{t} \mathfrak{f}+\widehat{p} \cdot \nabla_{x} \mathfrak{f}-\left[\left(\partial_{t} \phi+\widehat{p} \cdot \nabla_{x} \phi\right) p+\left(1+|p|^{2}\right)^{-1 / 2} \nabla_{x} \phi\right] \cdot \nabla_{p} \mathfrak{f}=0 .
\end{aligned}
$$

Here

$$
\widehat{p}=\frac{p}{\sqrt{1+|p|^{2}}}
$$

denotes the relativistic velocity of a particle with momentum $p$. The mass of each particle, the gravitational constant, and the speed of light are all normalized to one. A solution $(\mathfrak{f}, \phi)$ of this system is interpreted as follows: The spacetime is a Lorentzian manifold with a conformally flat metric which, in the coordinates $(t, x)$, takes the form

$$
g_{\mu \nu}=e^{2 \phi} \operatorname{diag}(-1,1,1,1) .
$$

The particle distribution $f$ defined on the mass shell in this metric is given by

$$
f(t, x, p)=\mathfrak{f}\left(t, x, e^{\phi} p\right) .
$$

The first mathematical study of this system was undertaken by Calogero in [19], where static solutions are analyzed and where also more details on the derivation of the system are given. Although the Nordström-Vlasov model of gravity does not describe physics correctly (in the classical limit the Vlasov-Poisson system of Newtonian gravity [21] is however obtained) it can nevertheless serve as a platform for developing new mathematical methods. The system has some common features with the Einstein-Vlasov system (see next Section 1.4) but seems in many respects to be less involved. The closest relationship from a mathematical point of view is the Vlasov-Maxwell system; this is evident in [23] where weak solutions are obtained in analogy with [35], in [22] where a continuation criterion for existence of classical solutions is established in analogy with [46] (an alternative approach is given in [76]), and in [62] where global existence in the case of two space dimensions is proved in analogy with [44]. The spherically symmetric case is studied in [6] and cannot be directly compared to the spherically symmetric Vlasov-Maxwell system (i.e. the VlasovPoisson system) since the hyperbolic nature of the equations is still present in the former system while it is lost in the latter case. In [6] it is shown that classical solutions exist globally in time for compactly supported initial data under the additional condition that there is a lower bound on the modulus of the angular momentum of the initial particle system. It should be noted that this is not a smallness condition and that the result holds for arbitrary large initial data satisfying this hypothesis.

Living Reviews in Relativity

http://www. livingreviews . org//rr-2005-2 


\subsection{The Einstein-Vlasov system}

In this section we will consider a self-gravitating collisionless gas and we will write down the Einstein-Vlasov system and describe its general mathematical features. Our presentation follows to a large extent the one by Rendall in [101]. We also refer to Ehlers [38] and Stewart [109] for more background on kinetic theory in general relativity.

Let $M$ be a four-dimensional manifold and let $g_{a b}$ be a metric with Lorentz signature $(-,+,+,+)$ so that $\left(M, g_{a b}\right)$ is a spacetime. We use the abstract index notation, which means that $g_{a b}$ is a geometric object and not the components of a tensor. See [117] for a discussion on this notation. The metric is assumed to be time-orientable so that there is a distinction between future and past directed vectors. The worldline of a particle with non-zero rest mass $m$ is a timelike curve and the unit future-directed tangent vector $v^{a}$ to this curve is the four-velocity of the particle. The four-momentum $p^{a}$ is given by $m v^{a}$. We assume that all particles have equal rest mass $m$ and we normalize so that $m=1$. One can also consider massless particles but we will rarely discuss this case. The possible values of the four-momentum are all future-directed unit timelike vectors and they constitute a hypersurface $P$ in the tangent bundle $T M$, which is called the mass shell. The distribution function $f$ that we introduced in the previous sections is a non-negative function on $P$. Since we are considering a collisionless gas, the particles travel along geodesics in spacetime. The Vlasov equation is an equation for $f$ that exactly expresses this fact. To get an explicit expression for this equation we introduce local coordinates on the mass shell. We choose local coordinates on $M$ such that the hypersurfaces $t=x^{0}=$ constant are spacelike so that $t$ is a time coordinate and $x^{j}, j=1,2,3$, are spatial coordinates (letters in the beginning of the alphabet always take values $0,1,2,3$ and letters in the middle take $1,2,3)$. A timelike vector is future directed if and only if its zero component is positive. Local coordinates on $P$ can then be taken as $x^{a}$ together with the spatial components of the four-momentum $p^{a}$ in these coordinates. The Vlasov equation then reads

$$
\partial_{t} f+\frac{p^{j}}{p^{0}} \partial_{x^{j}} f-\frac{1}{p^{0}} \Gamma_{a b}^{j} p^{a} p^{b} \partial_{p^{j}} f=0 .
$$

Here $a, b=0,1,2,3$ and $j=1,2,3$, and $\Gamma_{a b}^{j}$ are the Christoffel symbols. It is understood that $p^{0}$ is expressed in terms of $p^{j}$ and the metric $g_{a b}$ using the relation $g_{a b} p^{a} p^{b}=-1$ (recall that $m=1$ ).

In a fixed spacetime the Vlasov equation is a linear hyperbolic equation for $f$ and we can solve it by solving the characteristic system,

$$
\begin{aligned}
\frac{d X^{i}}{d s} & =\frac{P^{i}}{P^{0}}, \\
\frac{d P^{i}}{d s} & =-\Gamma_{a b}^{i} \frac{P^{a} P^{b}}{P^{0}} .
\end{aligned}
$$

In terms of initial data $f_{0}$ the solution to the Vlasov equation can be written as

$$
f\left(x^{a}, p^{i}\right)=f_{0}\left(X^{i}\left(0, x^{a}, p^{i}\right), P^{i}\left(0, x^{a}, p^{i}\right)\right),
$$

where $X^{i}\left(s, x^{a}, p^{i}\right)$ and $P^{i}\left(s, x^{a}, p^{i}\right)$ solve Equations $(26,27)$, and where $X^{i}\left(t, x^{a}, p^{i}\right)=x^{i}$ and $P^{i}\left(t, x^{a}, p^{i}\right)=p^{i}$.

In order to write down the Einstein-Vlasov system we need to define the energy-momentum tensor $T_{a b}$ in terms of $f$ and $g_{a b}$. In the coordinates $\left(x^{a}, p^{a}\right)$ on $P$ we define

$$
T_{a b}=-\int_{\mathbb{R}^{3}} f p_{a} p_{b}|g|^{1 / 2} \frac{d p^{1} d p^{2} d p^{3}}{p_{0}},
$$

where as usual $p_{a}=g_{a b} p^{b}$, and $|g|$ denotes the absolute value of the determinant of $g$. Equation (25) together with Einstein's equations,

$$
G_{a b}:=R_{a b}-\frac{1}{2} R g_{a b}=8 \pi T_{a b}+\Lambda g_{a b},
$$


then form the Einstein-Vlasov system. Here $G_{a b}$ is the Einstein tensor, $R_{a b}$ the Ricci tensor, $R$ is the scalar curvature and $\Lambda$ is the cosmological constant. In most of this review we will assume that $\Lambda=0$, but Section 2.3 is devoted to the case of non-vanishing cosmological constant (where also the case of adding a scalar field is discussed). We now define the particle current density

$$
N^{a}=-\int_{\mathbb{R}^{3}} f p^{a}|g|^{1 / 2} \frac{d p^{1} d p^{2} d p^{3}}{p_{0}} .
$$

Using normal coordinates based at a given point and assuming that $f$ is compactly supported it is not hard to see that $T_{a b}$ is divergence-free which is a necessary compatability condition since $G_{a b}$ is divergence-free by the Bianchi identities. A computation in normal coordinates also shows that $N^{a}$ is divergence-free, which expresses the fact that the number of particles is conserved. The definitions of $T_{a b}$ and $N^{a}$ immediately give us a number of inequalities. If $V^{a}$ is a future directed timelike or null vector then we have $N_{a} V^{a} \leq 0$ with equality if and only if $f=0$ at the given point. Hence $N^{a}$ is always future directed timelike if there are particles at that point. Moreover, if $V^{a}$ and $W^{a}$ are future directed timelike vectors then $T_{a b} V^{a} W^{b} \geq 0$, which is the dominant energy condition. If $X^{a}$ is a spacelike vector then $T_{a b} X^{a} X^{b} \geq 0$. This is called the non-negative pressure condition. These last two conditions together with the Einstein equations imply that $R_{a b} V^{a} V^{b} \geq 0$ for any timelike vector $V^{a}$, which is the strong energy condition. That the energy conditions hold for Vlasov matter is one reason that the Vlasov equation defines a well-behaved matter model in general relativity. Another reason is the well-posedness theorem by Choquet-Bruhat for the Einstein-Vlasov system that we will state below. Before stating that theorem we will first discuss the initial conditions imposed.

The data in the Cauchy problem for the Einstein-Vlasov system consist of the induced Riemannian metric $g_{i j}$ on the initial hypersurface $S$, the second fundamental form $k_{i j}$ of $S$ and matter data $f_{0}$. The relations between a given initial data set $\left(g_{i j}, k_{i j}\right)$ on a three-dimensional manifold $S$ and the metric $g_{i j}$ on the spacetime manifold is that there exists an embedding $\psi$ of $S$ into the spacetime such that the induced metric and second fundamental form of $\psi(S)$ coincide with the result of transporting $\left(g_{i j}, k_{i j}\right)$ with $\psi$. For the relation of the distribution functions $f$ and $f_{0}$ we have to note that $f$ is defined on the mass shell. The initial condition imposed is that the restriction of $f$ to the part of the mass shell over $\psi(S)$ should be equal to $f_{0} \circ\left(\psi^{-1}, d(\psi)^{-1}\right) \circ \phi$, where $\phi$ sends each point of the mass shell over $\psi(S)$ to its orthogonal projection onto the tangent space to $\psi(S)$. An initial data set for the Einstein-Vlasov system must satisfy the constraint equations, which read

$$
\begin{aligned}
R-k_{i j} k^{i j}+(\operatorname{tr} k)^{2} & =16 \pi \rho, \\
\nabla_{i} k_{l}^{i}-\nabla_{l}(\operatorname{tr} k) & =8 \pi j_{l} .
\end{aligned}
$$

Here $\rho=T_{a b} n^{a} n^{b}$ and $j^{a}=-h^{a b} T_{b c} n^{c}$, where $n^{a}$ is the future directed unit normal vector to the initial hypersurface and $h^{a b}=g^{a b}+n^{a} n^{b}$ is the orthogonal projection onto the tangent space to the initial hypersurface. In terms of $f_{0}$ we can express $\rho$ and $j^{l}$ by $\left(j^{a}\right.$ satisfies $n_{a} j^{a}=0$ so it can naturally be identified with a vector intrinsic to $S$ )

$$
\begin{aligned}
& \rho=\left.\left.\int_{\mathbb{R}^{3}} f p^{a} p_{a}\right|^{(3)} g\right|^{1 / 2} \frac{d p^{1} d p^{2} d p^{3}}{1+p_{j} p^{j}}, \\
& j_{l}=\left.\left.\int_{\mathbb{R}^{3}} f p_{l}\right|^{(3)} g\right|^{1 / 2} d p^{1} d p^{2} d p^{3} .
\end{aligned}
$$

Here $\left.\right|^{(3)} g \mid$ is the determinant of the induced Riemannian metric on $S$. We can now state the local existence theorem by Choquet-Bruhat [25] for the Einstein-Vlasov system.

Theorem 1 Let $S$ be a 3-dimensional manifold, $g_{i j}$ a smooth Riemannian metric on $S, k_{i j} a$ smooth symmetric tensor on $S$ and $f_{0}$ a smooth non-negative function of compact support on the

Living Reviews in Relativity

http://www. livingreviews. org/lrr-2005-2 
tangent bundle TS of S. Suppose that these objects satisfy the constraint equations (29, 30). Then there exists a smooth spacetime $\left(M, g_{a b}\right)$, a smooth distribution function $f$ on the mass shell of this spacetime, and a smooth embedding $\psi$ of $S$ into $M$ which induces the given initial data on $S$ such that $g_{a b}$ and $f$ satisfy the Einstein-Vlasov system and $\psi(S)$ is a Cauchy surface. Moreover, given any other spacetime $\left(M^{\prime}, g_{a b}^{\prime}\right)$, distribution function $f^{\prime}$ and embedding $\psi^{\prime}$ satisfying these conditions, there exists a diffeomorphism $\chi$ from an open neighbourhood of $\psi(S)$ in $M$ to an open neighbourhood of $\psi^{\prime}(S)$ in $M^{\prime}$ which satisfies $\chi \circ \psi=\psi^{\prime}$ and carries $g_{a b}$ and $f$ to $g_{a b}^{\prime}$ and $f^{\prime}$, respectively.

In this context we also mention that local existence has been proved for the Yang-MillsVlasov system in [26], and that this problem for the Einstein-Maxwell-Boltzmann system is treated in [11]. This result is however not complete, the non-negativity of $f$ is left unanswered. Also, the hypotheses on the scattering kernel in this work leave some room for further investigation. This problem concerning physically reasonable assumptions on the scattering kernel seems not well understood in the context of the Einstein-Boltzmann system, and a careful study of this issue would be desirable.

A main theme in the following sections is to discuss special cases for which the local existence theorem can be extended to a global one. There are interesting situations when this can be achieved, and such global existence theorems are not known for Einstein's equations coupled to other forms of phenomenological matter models, i.e. fluid models (see, however, [30]). In this context it should be stressed that the results in the previous sections show that the mathematical understanding of kinetic equations on a flat background space is well-developed. On the other hand the mathematical understanding of fluid equations on a flat background space (also in the absence of a Newtonian gravitational field) is not satisfying. It would be desirable to have a better mathematical understanding of these equations in the absence of gravity before coupling them to Einstein's equations. This suggests that the Vlasov equation is natural as matter model in mathematical general relativity. 


\section{Global Existence Theorems for the Einstein-Vlasov Sys- tem}

In general relativity two classes of initial data are distinguished. If an isolated matter distribution is studied, the data are called asymptotically flat. The initial hypersurface is topologically $\mathbb{R}^{3}$ and (since far away from the body one expects spacetime to be approximately flat) appropriate fall off conditions are imposed. Roughly, a smooth data set $\left(g_{i j}, k_{i j}, f_{0}\right)$ on $\mathbb{R}^{3}$ is said to be asymptotically flat if there exist global coordinates $x^{i}$ such that as $|x|$ tends to infinity the components $g_{i j}$ in these coordinates tend to $\delta_{i j}$, the components $k_{i j}$ tend to zero, $f_{0}$ has compact support and certain weighted Sobolev norms of $g_{i j}-\delta_{i j}$ and $k_{i j}$ are finite (see [101]). The symmetry classes that admit asymptotical flatness are few. The important ones are spherically symmetric and axially symmetric spacetimes. One can also consider a case in which spacetime is asymptotically flat except in one direction, namely cylindrical spacetimes. A lot of work has been done on the spherically symmetric case and this will be reviewed below. In the case of cylindrical symmetry it has been shown that if singularities form, then the first one must occur at the axis of symmetry [39].

Spacetimes that possess a compact Cauchy hypersurface are called cosmological spacetimes, and data are accordingly given on a compact 3-manifold. In this case the whole universe is modelled and not only an isolated body. In contrast to the asymptotically flat case, cosmological spacetimes admit a large number of symmetry classes. This gives one the possibility to study interesting special cases for which the difficulties of the full Einstein equations are strongly reduced. We will discuss below cases for which the spacetime is characterized by the dimension of its isometry group together with the dimension of the orbit of the isometry group.

\subsection{Spherically symmetric spacetimes}

The study of the global properties of solutions to the spherically symmetric Einstein-Vlasov system was initiated by Rein and Rendall in 1990. They chose to work in coordinates where the metric takes the form

$$
d s^{2}=-e^{2 \mu(t, r)} d t^{2}+e^{2 \lambda(t, r)} d r^{2}+r^{2}\left(d \theta^{2}+\sin ^{2} \theta d \varphi^{2}\right),
$$

where $t \in \mathbb{R}, r \geq 0, \theta \in[0, \pi], \varphi \in[0,2 \pi]$. These are called Schwarzschild coordinates. Asymptotic flatness is expressed by the boundary conditions

$$
\lim _{r \rightarrow \infty} \lambda(t, r)=\lim _{r \rightarrow \infty} \mu(t, r)=0, \quad \forall t \geq 0
$$

A regular centre is also required and is guaranteed by the boundary condition

$$
\lambda(t, 0)=0
$$

With

$$
x=(r \sin \phi \cos \theta, r \sin \phi \sin \theta, r \cos \phi)
$$

as spatial coordinate and

$$
v^{j}=p^{j}+\left(e^{\lambda}-1\right) \frac{x \cdot p}{r} \frac{x^{j}}{r}
$$

as momentum coordinates, the Einstein-Vlasov system reads

$$
\begin{aligned}
\partial_{t} f+e^{\mu-\lambda} \frac{v}{\sqrt{1+|v|^{2}}} \cdot \nabla_{x} f-\left(\lambda_{t} \frac{x \cdot v}{r}+e^{\mu-\lambda} \mu_{r} \sqrt{1+|v|^{2}}\right) \frac{x}{r} \cdot \nabla_{v} f & =0 \\
e^{-2 \lambda}\left(2 r \lambda_{r}-1\right)+1 & =8 \pi r^{2} \rho, \\
e^{-2 \lambda}\left(2 r \mu_{r}+1\right)-1 & =8 \pi r^{2} p .
\end{aligned}
$$

Living Reviews in Relativity

http: //www. livingreviews.org/lrr-2005-2 
The matter quantities are defined by

$$
\begin{aligned}
& \rho(t, x)=\int_{\mathbb{R}^{3}} \sqrt{1+|v|^{2}} f(t, x, v) d v \\
& p(t, x)=\int_{\mathbb{R}^{3}}\left(\frac{x \cdot v}{r}\right)^{2} f(t, x, v) \frac{d v}{\sqrt{1+|v|^{2}}} .
\end{aligned}
$$

Let us point out that this system is not the full Einstein-Vlasov system. The remaining field equations, however, can be derived from these equations. See [88] for more details. Let the square of the angular momentum be denoted by $L$, i.e.

$$
L:=|x|^{2}|v|^{2}-(x \cdot v)^{2} .
$$

A consequence of spherical symmetry is that angular momentum is conserved along the characteristics of Equation (31). Introducing the variable

$$
w=\frac{x \cdot v}{r},
$$

the Vlasov equation for $f=f(t, r, w, L)$ becomes

$$
\partial_{t} f+e^{\mu-\lambda} \frac{w}{E} \partial_{r} f-\left(\lambda_{t} w+e^{\mu-\lambda} \mu_{r} E-e^{\mu-\lambda} \frac{L}{r^{3} E}\right) \partial_{w} f=0,
$$

where

$$
E=E(r, w, L)=\sqrt{1+w^{2}+L / r^{2}} .
$$

The matter terms take the form

$$
\begin{aligned}
& \rho(t, r)=\frac{\pi}{r^{2}} \int_{-\infty}^{\infty} \int_{0}^{\infty} E f(t, r, w, L) d w d L, \\
& p(t, r)=\frac{\pi}{r^{2}} \int_{-\infty}^{\infty} \int_{0}^{\infty} \frac{w^{2}}{E} f(t, r, w, L) d w d L .
\end{aligned}
$$

Let us write down a couple of known facts about the system $(32,33,36,37,38)$. A solution to the Vlasov equation can be written as

$$
f(t, r, w, L)=f_{0}(R(0, t, r, w, L), W(0, t, r, w, L), L),
$$

where $R$ and $W$ are solutions to the characteristic system

$$
\begin{aligned}
\frac{d R}{d s} & =e^{(\mu-\lambda)(s, R)} \frac{W}{E(R, W, L)}, \\
\frac{d W}{d s} & =-\lambda_{t}(s, R) W-e^{(\mu-\lambda)(s, R)} \mu_{r}(s, R) E(R, W, L)+e^{(\mu-\lambda)(s, R)} \frac{L}{R^{3} E(R, W, L)},
\end{aligned}
$$

such that the trajectory $(R(s, t, r, w, L), W(s, t, r, w, L), L)$ goes through the point $(r, w, L)$ when $s=t$. This representation shows that $f$ is non-negative for all $t \geq 0$ and that $f \leq\left\|f_{0}\right\|_{\infty}$. There are two known conservation laws for the Einstein-Vlasov system: conservation of the number of particles,

$$
4 \pi^{2} \int_{0}^{\infty} e^{\lambda(t, r)}\left(\int_{-\infty}^{\infty} \int_{0}^{\infty} f(t, r, w, L) d L d w\right) d r
$$

and conservation of the ADM mass

$$
M:=4 \pi \int_{0}^{\infty} r^{2} \rho(t, r) d r .
$$


Let us now review the global results concerning the Cauchy problem that have been proved for the spherically symmetric Einstein-Vlasov system. As initial data we take a spherically symmetric, non-negative, and continuously differentiable function $f_{0}$ with compact support that satisfies

$$
4 \pi^{2} \int_{0}^{r} \int_{-\infty}^{\infty} \int_{0}^{\infty} E f_{0}(r, w, L) d w d L d r<\frac{r}{2} .
$$

This condition guarantees that no trapped surfaces are present initially. In [88] it is shown that for such an initial datum there exists a unique, continuously differentiable solution $f$ with $f(0)=f_{0}$ on some right maximal interval $[0, T)$. If the solution blows up in finite time, i.e. if $T<\infty$, then $\rho(t)$ becomes unbounded as $t \rightarrow T$. Moreover, a continuation criterion is shown that says that a local solution can be extended to a global one provided the $v$-support of $f$ can be bounded on $[0, T)$. (In [88] they chose to work in the momentum variable $v$ rather than $w, L$.) This is analogous to the situation for the Vlasov-Maxwell system where the function $Q(t)$ was introduced for the $v$-support. A control of the $v$-support immediately implies that $\rho$ and $p$ are bounded in view of Equations (34, 35). In the Vlasov-Maxwell case the field equations have a regularizing effect in the sense that derivatives can be expressed through spatial integrals, and it follows [46] that the derivatives of $f$ also can be bounded if the $v$-support is bounded. For the Einstein-Vlasov system such a regularization is less clear, since e.g. $\mu_{r}$ depends on $\rho$ in a pointwise manner. However, certain combinations of second and first order derivatives of the metric components can be expressed in terms of matter components only, without derivatives (a consequence of the geodesic deviation equation). This fact turns out to be sufficient for obtaining bounds also on the derivatives of $f$ (see [88] for details). By considering initial data sufficiently close to zero, Rein and Rendall show that the $v$-support is bounded on $[0, T)$, and the continuation criterion then implies that $T=\infty$. It should be stressed that even for small data no global existence result like this one is known for any other phenomenological matter model coupled to Einstein's equations. The resulting spacetime in [88] is geodesically complete, and the components of the energy momentum tensor as well as the metric quantities decay with certain algebraic rates in $t$. The mathematical method used by Rein and Rendall is inspired by the analogous small data result for the Vlasov-Poisson equation by Bardos and Degond [12]. This should not be too surprising since for small data the gravitational fields are expected to be small and a Newtonian spacetime should be a fair approximation. In this context we point out that in [89] it is proved that the Vlasov-Poisson system is indeed the nonrelativistic limit of the spherically symmetric Einstein-Vlasov system, i.e. the limit when the speed of light $c \rightarrow \infty$. (In [96] this issue is studied in the asymptotically flat case without symmetry assumptions.) Finally, we mention that there is an analogous small data result using a maximal time coordinate [101] instead of a Schwarzschild time coordinate. In these coordinates trapped surfaces are allowed in contrast to the Schwarzschild coordinates.

The case with general data is more subtle. Rendall has shown [95] that there exist data leading to singular spacetimes as a consequence of Penrose's singularity theorem. This raises the question of what we mean by global existence for such data. The Schwarzschild time coordinate is expected to avoid the singularity, and by global existence we mean that solutions remain smooth as Schwarzschild time tends to infinity. Even though spacetime might be only partially covered in Schwarzschild coordinates, a global existence theorem for general data would nevertheless be very important since it is likely that it would constitute a central step for proving weak cosmic censorship. Indeed, if this coordinate system can be shown to cover the domain of outer communications and if null infinity could be shown to be complete, then weak cosmic censorship would follow. A partial investigation for general data in Schwarzschild coordinates was done in [92] and in maximal-isotropic coordinates in [101]. In Schwarzschild coordinates it is shown that if singularities form in finite time the first one must be at the centre. More precisely, if $f(t, r, w, L)=0$ when $r>\epsilon$ for some $\epsilon>0$, and for all $t, w$, and $L$, then the solution remains smooth for all time. This rules out singularities of the shell-crossing type, which can be an annoying problem for other

Living Reviews in Relativity

http://www . livingreviews . org//rr-2005-2 
matter models (e.g. dust). The main observation in [92] is a cancellation property in the term

$$
\lambda_{t} w+e^{\mu-\lambda} \mu_{r} E
$$

in the characteristic equation (41). In [101] a similar result is shown, but here also an assumption that one of the metric functions is bounded at the centre is assumed. However, with this assumption the result follows in a more direct way and the analysis of the Vlasov equation is not necessary, which indicates that such a result might be true more generally. Recently, Dafermos and Rendall [33] have shown a similar result for the Einstein-Vlasov system in double-null coordinates. The main motivation for studying the system in these coordinates has its origin from the method of proof of the cosmic censorship conjecture for the Einstein-scalar field system by Christodoulou [31]. An essential part of his method is based on the understanding of the formation of trapped surfaces [28]. The presence of trapped surfaces (for the relevant initial data) is then crucial in proving the completeness of future null infinity in [31]. In [32] the relation between trapped surfaces and the completeness of null infinity was strengthened; a single trapped surface or marginally trapped surface in the maximal development implies completeness of null infinty. The theorem holds true for any spherically symmetric matter spacetime if the matter model is such that "first" singularities necessarily emanate from the center (where the notion of "first" is tied to the casual structure). The results in [92] and in [101] are not sufficient for concluding that the hypothesis of the matter needed in the theorem in [32] is satisfied, since they concern a portion of the maximal development covered by particular coordinates. Therefore, Dafermos and Rendall [33] choose double-null coordinates which cover the maximal development, and they show that the mentioned hypothesis is satisfied for Vlasov matter.

In [93] a numerical study was undertaken of the Einstein-Vlasov system in Schwarzschild coordinates. A numerical scheme originally used for the Vlasov-Poisson system was modified to the spherically symmetric Einstein-Vlasov system. It has been shown by Rein and Rodewis [94] that the numerical scheme has the desirable convergence properties. (In the Vlasov-Poisson case convergence was proved in [106]; see also [40]). The numerical experiments support the conjecture that solutions are singularity-free. This can be seen as evidence that weak cosmic censorship holds for collisionless matter. It may even hold in a stronger sense than in the case of a massless scalar field (see [29, 31]). There may be no naked singularities formed for any regular initial data rather than just for generic data. This speculation is based on the fact that the naked singularities that occur in scalar field collapse appear to be associated with the existence of type II critical collapse, while Vlasov matter is of type I. This is indeed the primary goal of their numerical investigation: to analyze critical collapse and decide whether Vlasov matter is type I or type II.

These different types of matter are defined as follows. Given small initial data no black holes are expected to form and matter will disperse (which has been proved for a scalar field [27] and for Vlasov matter [88]). For large data, black holes will form and consequently there is a transition regime separating dispersion of matter and formation of black holes. If we introduce a parameter $A$ on the initial data such that for small $A$ dispersion occurs and for large $A$ a black hole is formed, we get a critical value $A_{\mathrm{c}}$ separating these regions. If we take $A>A_{\mathrm{c}}$ and denote by $m_{\mathrm{B}}(A)$ the mass of the black hole, then if $m_{\mathrm{B}}(A) \rightarrow 0$ as $A \rightarrow A_{\mathrm{c}}$ we have type II matter, whereas for type I matter this limit is positive and there is a mass gap. For more information on critical collapse we refer to the review paper by Gundlach [50].

For Vlasov matter there is an independent numerical simulation by Olabarrieta and Choptuik [75] (using a maximal time coordinate) and their conclusion agrees with the one in [93]. Critical collapse is related to self similar solutions; Martín-García and Gundlach [68] have presented a construction of such solutions for the massless Einstein-Vlasov system by using a method based partially on numerics. Since such solutions often are related to naked singularities, it is important to note that their result is for the massless case (in which case there is no known analogous result to the small data theorem in [88]) and their initial data are not in the class that we have 
described above.

We end this section with a discussion of the spherically symmetric Einstein-Vlasov-Maxwell system, i.e. the case considered above with charged particles. Whereas the constraint equations in the uncharged case do not involve any problems to solve once the distribution function is given (and satisfies Equation (43)), the charged case is more challenging. In [73] it is shown that solutions to the constraint equations do exist for the Einstein-Vlasov-Maxwell system. In [72] local existence is then shown together with a continuation criterion, and in [71] the regularity theorem in [92] is generalized to this case.

\subsection{Cosmological solutions}

In cosmology the whole universe is modelled, and the "particles" in the kinetic description are galaxies or even clusters of galaxies. The main goal again is to determine the global properties of the solutions to the Einstein-Vlasov system. In order to do so, a global time coordinate $t$ must be found (global existence) and the asymptotic behaviour of the solutions when $t$ tends to its limiting values has to be analyzed. This might correspond to approaching a singularity (e.g. the big bang singularity) or to a phase of unending expansion. Since the general case is beyond the range of current mathematical techniques, all known results are for cases with symmetry (see however [9] where to some extent global properties are established in the case without symmetry).

There are several existing results on global time coordinates for solutions of the Einstein-Vlasov system. In the spatially homogeneous case it is natural to choose a Gaussian time coordinate based on a homogeneous hypersurface. The maximal range of a Gaussian time coordinate in a solution of the Einstein-Vlasov system evolving from homogenous data on a compact manifold was determined in [98]. The range is finite for models of Bianchi IX and Kantowski-Sachs types. It is finite in one time direction and infinite in the other for the other Bianchi types. The asymptotic behaviour of solutions in the spatially homogeneous case has been analyzed in [103] and [104]. In [103], the case of massless particles is considered, whereas the massive case is studied in [104]. Both the nature of the initial singularity and the phase of unlimited expansion are analyzed. The main concern is the behaviour of Bianchi models I, II, and III. The authors compare their solutions with the solutions to the corresponding perfect fluid models. A general conclusion is that the choice of matter model is very important since for all symmetry classes studied there are differences between the collisionless model and a perfect fluid model, both regarding the initial singularity and the expanding phase. The most striking example is for the Bianchi II models, where they find persistent oscillatory behaviour near the singularity, which is quite different from the known behaviour of Bianchi type II perfect fluid models. In [104] it is also shown that solutions for massive particles are asymptotic to solutions with massless particles near the initial singularity. For Bianchi I and II it is also proved that solutions with massive particles are asymptotic to dust solutions at late times. It is conjectured that the same holds true also for Bianchi III. This problem is then settled by Rendall in [102].

All other results presently available on the subject concern spacetimes that admit a group of isometries acting on two-dimensional spacelike orbits, at least after passing to a covering manifold. The group may be two-dimensional (local $U(1) \times U(1)$ or $T^{2}$ symmetry) or three-dimensional (spherical, plane, or hyperbolic symmetry). In all these cases, the quotient of spacetime by the symmetry group has the structure of a two-dimensional Lorentzian manifold $Q$. The orbits of the group action (or appropriate quotients in the case of a local symmetry) are called surfaces of symmetry. Thus, there is a one-to-one correspondence between surfaces of symmetry and points of $Q$. There is a major difference between the cases where the symmetry group is two- or threedimensional. In the three-dimensional case no gravitational waves are admitted, in contrast to the two-dimensional case. In the former case, the field equations reduce to ODEs while in the latter their evolution part consists of nonlinear wave equations. Three types of time coordinates that

Living Reviews in Relativity

http://www. livingreviews . org//rr-2005-2 
have been studied in the inhomogeneous case are CMC, areal, and conformal coordinates. A CMC time coordinate $t$ is one where each hypersurface of constant time has constant mean curvature $(\mathrm{CMC})$ and on each hypersurface of this kind the value of $t$ is the mean curvature of that slice. In the case of areal coordinates, the time coordinate is a function of the area of the surfaces of symmetry (e.g. proportional to the area or proportional to the square root of the area). In the case of conformal coordinates, the metric on the quotient manifold $Q$ is conformally flat.

Let us first consider spacetimes $(M, g)$ admitting a three-dimensional group of isometries. The topology of $M$ is assumed to be $\mathbb{R} \times S^{1} \times F$, with $F$ a compact two-dimensional manifold. The universal covering $\hat{F}$ of $F$ induces a spacetime $(\hat{M}, \hat{g})$ by $\hat{M}=\mathbb{R} \times S^{1} \times \hat{F}$ and $\hat{g}=p^{*} g$, where $p: \hat{M} \rightarrow M$ is the canonical projection. A three-dimensional group $G$ of isometries is assumed to act on $(\hat{M}, \hat{g})$. If $F=S^{2}$ and $G=S O(3)$, then $(M, g)$ is called spherically symmetric; if $F=T^{2}$ and $G=E_{2}$ (Euclidean group), then $(M, g)$ is called plane symmetric; and if $F$ has genus greater than one and the connected component of the symmetry group $G$ of the hyperbolic plane $H^{2}$ acts isometrically on $\hat{F}=H^{2}$, then $(M, g)$ is said to have hyperbolic symmetry.

In the case of spherical symmetry the existence of one compact CMC hypersurface implies that the whole spacetime can be covered by a CMC time coordinate that takes all real values [97, 18]. The existence of one compact CMC hypersurface in this case was proved later by Henkel [52] using the concept of prescribed mean curvature (PMC) foliation. Accordingly this gives a complete picture in the spherically symmetric case regarding CMC foliations. In the case of areal coordinates, Rein [82] has shown, under a size restriction on the initial data, that the past of an initial hypersurface can be covered. In the future direction it is shown that areal coordinates break down in finite time.

In the case of plane and hyperbolic symmetry, Rendall and Rein showed in [97] and [82], respectively, that the existence results (for CMC time and areal time) in the past direction for spherical symmetry also hold for these symmetry classes. The global CMC foliation results to the past imply that the past singularity is a crushing singularity, since the mean curvature blows up at the singularity. In addition, Rein also proved in his special case with small initial data that the Kretschmann curvature scalar blows up when the singularity is approached. Hence, the singularity is both a crushing and a curvature singularity in this case. In both of these works the question of global existence to the future was left open. This gap was closed in [7], and global existence to the future was established in both CMC and areal coordinates. The global existence result for CMC time is partly a consequence of the global existence theorem in areal coordinates, together with a theorem by Henkel [52] that shows that there exists at least one hypersurface with (negative) constant mean curvature. Also, the past direction was analyzed in areal coordinates and global existence was shown without any smallness condition on the data. It is, however, not concluded if the past singularity in this more general case without the smallness condition on the data is a curvature singularity as well. The question whether the areal time coordinate, which is positive by definition, takes all values in the range $(0, \infty)$ or only in $\left(R_{0}, \infty\right)$ for some positive $R_{0}$ is also left open. In the special case in [82], it is indeed shown that $R_{0}=0$, but there is an example for vacuum spacetimes in the more general case of $U(1) \times U(1)$ symmetry where $R_{0}>0$. This question was resolved by Weaver [118]. She proves that if spacetime contains Vlasov matter (i.e. $f \neq 0$ ) then $R=0$. Her result applies to a more general case which we now turn to.

For spacetimes admitting a two-dimensional isometry group, the first study was done by Rendall [100] in the case of local $U(1) \times U(1)$ symmetry (or local $T^{2}$ symmetry). For a discussion of the topologies of these spacetimes we refer to the original paper. In the model case the spacetime is topologically of the form $\mathbb{R} \times T^{3}$, and to simplify our discussion later on we write down the metric in areal coordinates for this type of spacetime:

$$
\begin{aligned}
g= & e^{2(\eta-U)}\left(-\alpha d t^{2}+d \theta^{2}\right)+e^{-2 U} t^{2}[d y+H d \theta+M d t]^{2} \\
& +e^{2 U}[d x+A d y+(G+A H) d \theta+(L+A M) d t]^{2} .
\end{aligned}
$$


Here the metric coefficients $\eta, U, \alpha, A, H, L$, and $M$ depend on $t$ and $\theta$ and $\theta, x, y \in S^{1}$. In [100] $\mathrm{CMC}$ coordinates are considered rather than areal coordinates. The $\mathrm{CMC}$ and the areal coordinate foliations are both geometrically based time foliations. The advantage with a CMC approach is that the definition of a CMC hypersurface does not depend on any symmetry assumptions and it is possible that $\mathrm{CMC}$ foliations will exist for rather general spacetimes. The areal coordinate foliation, on the other hand, is adapted to the symmetry of spacetime but it has analytical advantages that we will see below.

Under the hypothesis that there exists at least one CMC hypersurface, Rendall proves, without any smallness condition on the data, that the past of the given CMC hypersurface can be globally foliated by CMC hypersurfaces and that the mean curvature of these hypersurfaces blows up at the past singularity. Again, the future direction was left open. The result in [100] holds for Vlasov matter and for matter described by a wave map (which is not a phenomenological matter model). That the choice of matter model is important was shown by Rendall [99] who gives a non-global existence result for dust, which leads to examples of spacetimes [59] that are not covered by a CMC foliation.

There are several possible subcases to the $U(1) \times U(1)$ symmetry class. The plane case where the symmetry group is three-dimensional is one subcase and the form of the metric in areal coordinates is obtained by letting $A=G=H=L=M=0$ and $U=\log t / 2$ in Equation (44). Another subcase, which still admits only two Killing fields (and which includes plane symmetry as a special case), is Gowdy symmetry. It is obtained by letting $G=H=L=M=0$ in Equation (44). In [4], the author considers Gowdy symmetric spacetimes with Vlasov matter. It is proved that the entire maximal globally hyperbolic spacetime can be foliated by constant areal time slices for arbitrary (in size) initial data. The areal coordinates are used in a direct way for showing global existence to the future whereas the analysis for the past direction is carried out in conformal coordinates. These coordinates are not fixed to the geometry of spacetime and it is not clear that the entire past has been covered. A chain of geometrical arguments then shows that areal coordinates indeed cover the entire spacetime. This method was applied to the problem on hyperbolic and plane symmetry in [7]. The method in [4] was in turn inspired by the work [16] for vacuum spacetimes where the idea of using conformal coordinates in the past direction was introduced. As pointed out in [7], the result by Henkel [53] guarantees the existence of one CMC hypersurface in the Gowdy case and, together with the global areal foliation in [4], it follows that Gowdy spacetimes with Vlasov matter can be globally covered by CMC hypersurfaces as well (also to the future). The general case of $U(1) \times U(1)$ symmetry was considered in [8], where it is shown that there exist global CMC and areal time foliations which complete the picture. In this result as well as in the preceeding subcases mentioned above the question whether or not the areal time coordinate takes values in $(0, \infty)$ or in $(R, \infty), R>0$, was left open. This issue was solved by Weaver in [118] where she concludes that $R=0$ if the distribution function is not identically zero initially.

A number of important questions remain open. To analyze the nature of the initial singularity, which at present is known only for small initial data in the case considered in [82], would be very interesting. The question of the asymptotics in the future direction is also an important issue where very little is known. The only situation where a result has been obtained is in the case with hyperbolic symmetry. Under a certain size restriction on the initial data, Rein [87] shows future geodesic completeness. However, in models with a positive cosmological constant more can be said.

\subsection{Cosmological models with a cosmological constant or a scalar field}

The present cosmological observations indicate that the expansion of the universe is accelerating, and this has influenced the theoretical studies in the field during the last years. One way to produce models with accelerated expansion is to choose a positive cosmological constant in the Einstein equations. Another way is to include a nonlinear scalar field among the matter fields. In

Living Reviews in Relativity

http://www. livingreviews.org//rr-2005-2 
this section we will review the recent results for the Einstein-Vlasov system where a cosmological constant, or a linear or nonlinear scalar field have been included into the model.

As in the previous section we start with the models with highest degree of symmetry, i.e. the locally spatially homogeneous models. In the case of a positive cosmological constant Lee [63] has shown global existence as well as future causal geodesic completeness for initial data which have Bianchi symmetry. She also obtains the time decay of the components of the energy momentum tensor as $t \rightarrow \infty$. The past direction for some spatially homogeneous models is considered in [111]. Existence back to the initial singularity is proved and the case with a negative cosmological constant is discussed. In [64] Lee considers the case with a nonlinear scalar field coupled to Vlasov matter. The form of the energy momentum then reads

$$
T_{\alpha \beta}=T_{\alpha \beta}^{\text {Vlasov }}+\nabla_{\alpha} \phi \nabla_{\beta} \phi-\left(\frac{1}{2} \nabla^{\gamma} \phi \nabla_{\gamma} \phi+V(\phi)\right) g_{\alpha \beta} .
$$

Here $\phi$ is the scalar field and $V$ is a potential, and the Bianchi identities lead to the following equation for the scalar field:

$$
\nabla^{\gamma} \phi \nabla_{\gamma} \phi=V^{\prime}(\phi) .
$$

Under the assumption that $V$ is non-negative and $C^{2}$, global existence to the future is obtained and if the potential is restricted to the form

$$
V(\phi)=V_{0} e^{-c \Phi},
$$

where $0<c<4 \sqrt{\pi}$ then future geodesic completeness is proved.

In the previous Section 2.3 we discussed the situation when spacetime admits a three-dimensional group of isometries and we distinguished three cases: plane, spherical, and hyperbolic symmetry. In area time coordinates the metric takes the form

$$
d s^{2}=-e^{\mu(t, r)} d t^{2}+e^{\lambda(t, r)} d r^{2}+t^{2}\left(d \theta^{2}+\sin _{\kappa}^{2} \theta d \varphi^{2}\right),
$$

where $k=0,-1,+1$ correspond to the plane, spherical, and hyperbolic case, respectively, and where $\sin _{0} \theta=1, \sin _{1} \theta=\sin \theta$, and $\sin _{-1} \theta=\sinh \theta$. In [113] the Einstein-Vlasov system with a positive cosmological constant is investigated in the future (expanding) direction in the case of plane and hyperbolic symmetry. The authors prove global existence to the future in these coordinates and they also show future geodesic completeness. The positivity of the cosmological constant is crucial for the latter result. Recall that in the case of $\Lambda=0$, future geodesic completenss has only been established for hyperbolic symmetry under a smallness condition of the initial data [87]. Finally a form of the cosmic no-hair conjecture is obtained in [113] for this class of spacetimes. Indeed, here it is shown that the de Sitter solution acts as a model for the dynamics of the solutions by proving that the generalized Kasner exponents tend to $1 / 3$ as $t \rightarrow \infty$, which in the plane case is the de Sitter solution. The remaining case of spherical symmetry is analyzed in [112]. Recall that when $\Lambda=0$, Rein [82] showed that solutions can only exist for finite time in the future direction in area time coordinates. By adding a positive cosmological constant, global existence to the future is shown to hold true if initial data is given on $t=t_{0}$, where $t_{0}^{2}>1 / \Lambda$. The asymptotic behaviour of the matter terms is also investigated and slightly stronger decay estimates are obtained in this case compared to the case of plane and hyperbolic symmetry.

The results discussed so far in this section have concerned the future time direction and a positive cosmological constant. The past direction with a negative cosmological constant is analyzed in [111], where it is shown that for plane and spherical symmetry the areal time coordinate takes all positive values, which is in analogy with Weaver's [118] result for $\Lambda=0$. If initial data are restricted by a smallness condition the theorem is proven also in the hyperbolic case, and for such data the result of the theorem holds true in all of the three symmetry classes when the cosmological 
constant is positive. The early-time asymptotics in the case of small initial data is also analyzed and is shown to be Kasner-like.

In [114] the Einstein-Vlasov system with a linear scalar field is analyzed in the case of plane, spherical, and hyperbolic symmetry. Here the potential $V$ in Equations $(45,46)$ is zero. A local existence theorem and a continuation criterion, involving bounds on derivatives of the scalar field in addition to a bound on the support of one of the moment variables, is proven. For the Einstein scalar field system, i.e. when $f=0$, the continuation criterion is shown to be satisfied in the future direction and global existence follows in that case.

Living Reviews in Relativity

http: //www . livingreviews . org/lrr-2005-2 


\section{Stationary Solutions to the Einstein-Vlasov System}

Equilibrium states in galactic dynamics can be described as stationary solutions of the EinsteinVlasov system, or of the Vlasov-Poisson system in the Newtonian case. Here we will consider the former case for which only static, spherically symmetric solutions have been constructed, but we mention that in the latter case also, stationary axially symmetric solutions have been found by Rein [85].

In the static, spherically symmetric case, the problem can be formulated as follows. Let the spacetime metric have the form

$$
d s^{2}=-e^{2 \mu(r)} d t^{2}+e^{2 \lambda(r)} d r^{2}+r^{2}\left(d \theta^{2}+\sin ^{2} \theta d \varphi^{2}\right),
$$

where $r \geq 0, \theta \in[0, \pi], \varphi \in[0,2 \pi]$. As before, asymptotic flatness is expressed by the boundary conditions

$$
\lim _{r \rightarrow \infty} \lambda(r)=\lim _{r \rightarrow \infty} \mu(r)=0, \quad \forall t \geq 0,
$$

and a regular centre requires

$$
\lambda(0)=0
$$

Following the notation in Section 2.1, the time-independent Einstein-Vlasov system reads

$$
\begin{aligned}
e^{\mu-\lambda} \frac{v}{\sqrt{1+|v|^{2}}} \cdot \nabla_{x} f-\sqrt{1+|v|^{2}} e^{\mu-\lambda} \mu_{r} \frac{x}{r} \cdot \nabla_{v} f & =0, \\
e^{-2 \lambda}\left(2 r \lambda_{r}-1\right)+1 & =8 \pi r^{2} \rho, \\
e^{-2 \lambda}\left(2 r \mu_{r}+1\right)-1 & =8 \pi r^{2} p .
\end{aligned}
$$

The matter quantities are defined as before:

$$
\begin{aligned}
& \rho(x)=\int_{\mathbb{R}^{3}} \sqrt{1+|v|^{2}} f(x, v) d v \\
& p(x)=\int_{\mathbb{R}^{3}}\left(\frac{x \cdot v}{r}\right)^{2} f(x, v) \frac{d v}{\sqrt{1+|v|^{2}}} .
\end{aligned}
$$

The quantities

$$
E:=e^{\mu(r)} \sqrt{1+|v|^{2}}, \quad L=|x|^{2}|v|^{2}-(x \cdot v)^{2}=|x \times v|^{2}
$$

are conserved along characteristics. $E$ is the particle energy and $L$ is the angular momentum squared. If we let

$$
f(x, v)=\Phi(E, L)
$$

for some function $\Phi$, the Vlasov equation is automatically satisfied. The form of $\Phi$ is usually restricted to

$$
\Phi(E, L)=\phi(E)\left(L-L_{0}\right)^{l}
$$

where $l>-1 / 2$ and $L_{0} \geq 0$. If $\phi(E)=\left(E-E_{0}\right)_{+}^{k}, k>-1$, for some positive constant $E_{0}$, this is called the polytropic ansatz. The case of isotropic pressure is obtained by letting $l=0$ so that $\Phi$ only depends on $E$. We refer to [81] for information on the role of $L_{0}$.

In passing, we mention that for the Vlasov-Poisson system it has been shown [15] that every static spherically symmetric solution must have the form $f=\Phi(E, L)$. This is referred to as Jeans' theorem. It was an open question for some time to decide whether or not this was also true for the Einstein-Vlasov system. This was settled in 1999 by Schaeffer [107], who found solutions that do not have this particular form globally on phase space, and consequently, Jeans' theorem is not valid in the relativistic case. However, almost all results in this field rest on this ansatz. By inserting 
the ansatz for $f$ in the matter quantities $\rho$ and $p$, a nonlinear system for $\lambda$ and $\mu$ is obtained, in which

$$
\begin{aligned}
& e^{-2 \lambda}\left(2 r \lambda_{r}-1\right)+1=8 \pi r^{2} G_{\Phi}(r, \mu), \\
& e^{-2 \lambda}\left(2 r \mu_{r}+1\right)-1=8 \pi r^{2} H_{\Phi}(r, \mu),
\end{aligned}
$$

where

$$
\begin{aligned}
& G_{\Phi}(r, \mu)=\frac{2 \pi}{r^{2}} \int_{1}^{\infty} \int_{0}^{r^{2}\left(\epsilon^{2}-1\right)} \Phi\left(e^{\mu(r)} \epsilon, L\right) \frac{\epsilon^{2}}{\sqrt{\epsilon^{2}-1-L / r^{2}}} d L d \epsilon, \\
& H_{\Phi}(r, \mu)=\frac{2 \pi}{r^{2}} \int_{1}^{\infty} \int_{0}^{r^{2}\left(\epsilon^{2}-1\right)} \Phi\left(e^{\mu(r)} \epsilon, L\right) \sqrt{\epsilon^{2}-1-L / r^{2}} d L d \epsilon .
\end{aligned}
$$

Existence of solutions to this system was first proved in the case of isotropic pressure in [90] and then extended to the general case in [81]. The main problem is then to show that the resulting solutions have finite (ADM) mass and compact support. This is accomplished in [90] for a polytropic ansatz with isotropic pressure and in [81] for a polytropic ansatz with possible anisotropic pressure. They use a perturbation argument based on the fact that the Vlasov-Poisson system is the limit of the Einstein-Vlasov system as the speed of light tends to infinity [89]. Two types of solutions are constructed, those with a regular centre [90, 81], and those with a Schwarzschild singularity in the centre [81]. In [91] Rendall and Rein go beyond the polytropic ansatz and assume that $\Phi$ satisfies

$$
\Phi(E, L)=c\left(E-E_{0}\right)_{+}^{k} L^{l}+O\left(\left(E_{0}-E\right)_{+}^{\delta+k}\right) L^{l} \quad \text { as } E \rightarrow E_{0},
$$

where $k>-1, l>-1 / 2, k+l+1 / 2>0, k<l+3 / 2$. They show that this assumption is sufficient for obtaining steady states with finite mass and compact support. The result is obtained in a more direct way and is not based on the perturbation argument mentioned above. Their method is inspired by a work on stellar models by Makino [67], in which he considers steady states of the Euler-Einstein system. In [91] there is also an interesting discussion about steady states that appear in the astrophysics literature. They show that their result applies to most of these steady states, which proves that they have the desirable property of finite mass and compact support.

All solutions described so far have the property that the support of $\rho$ contains a ball about the centre. In [84] Rein shows that there exist steady states whose support is a finite, spherically symmetric shell, so that they have a vacuum region in the centre.

At present, there are almost no known results concerning the stability properties of the steady states to the Einstein-Vlasov system. In the Vlasov-Poisson case, however, the nonlinear stability of stationary solutions has been investigated by Guo and Rein [51] using the energy-Casimir method. In the Einstein-Vlasov case, Wolansky [121] has applied the energy-Casimir method and obtained some insights but the theory in this case is much less developed than in the Vlasov-Poisson case and the stability problem is essentially open.

Living Reviews in Relativity

http://www. livingreviews.org/lrr-2005-2 


\section{Acknowledgements}

I would like to thank Alan Rendall for helpful suggestions. 


\section{References}

[1] Andréasson, H., "Controlling the propagation of the support for the relativistic Vlasov equation with a selfconsistent Lorentz invariant field", Indiana Univ. Math. J., 45, 617-642, (1996). $\quad 1.2$

[2] Andréasson, H., "Regularity of the gain term and strong $L^{1}$ convergence to equilibrium for the relativistic Boltzmann equation", SIAM J. Math. Anal., 27, 1386-1405, (1996). 1.1, 1.1

[3] Andréasson, H., "Global existence of smooth solutions in three dimensions for the semiconductor Vlasov-Poisson-Boltzmann equation", Nonlinear Anal., 28, 1193-1211, (1997). 1.2

[4] Andréasson, H., "Global foliations of matter spacetimes with Gowdy symmetry", Commun. Math. Phys., 206, 337-366, (1999). 2.2

[5] Andréasson, H., Calogero, S., and Illner, R., "On Blowup for Gain-Term-Only classical and relativistic Boltzmann equations", submitted. To appear in Math. Method. Appl. Sci. 1.1

[6] Andréasson, H., Calogero, S., and Rein, G., "Global classical solutions to the spherically symmetric Nordström-Vlasov system", submitted. To appear in Math. Proc. Camb. Phil. Soc. 1.3

[7] Andréasson, H., Rein, G., and Rendall, A.D., "On the Einstein-Vlasov system with hyperbolic symmetry", Math. Proc. Camb. Phil. Soc., 134, 529-549, (2003). 2.2, 2.2

[8] Andréasson, H., Rendall, A.D., and Weaver, M., "Existence of CMC and Constant Areal Time Foliations in $T^{2}$ Symmetric Spacetimes with Vlasov Matter", Commun. Part. Diff. Eq., 29, 237-262, (2004). 2.2

[9] Anguige, K., "Isotropic cosmological singularities 3: The Cauchy problem for the inhomogeneous conformal Einstein-Vlasov equations", Ann. Phys. (N.Y.), 282, 395-419, (2000). 2.2

[10] Arkeryd, L., "On the strong $L^{1}$ trend to equilibrium for the Boltzmann equation", Stud. Appl. Math., 87, 283-288, (1992). 1.1

[11] Bancel, D., and Choquet-Bruhat, Y., "Existence, uniqueness and local stability for the Einstein-Maxwell-Boltzmann system”, Commun. Math. Phys., 33, 83-96, (1993). 1.4

[12] Bardos, C., and Degond, P., "Global existence for the Vlasov-Poisson equation in three space variables with small initial data", Ann. Inst. Henri Poincare, 2, 101-118, (1985). 1.2, 2.1

[13] Bardos, C., Degond, P., and Ha, T.N., "Existence globale des solutions des équations de Vlasov-Poisson relativistes en dimension 3", C. R. Acad. Sci., 301, 265-268, (1985). 1.2

[14] Batt, J., "Global symmetric solutions of the initial value problem of stellar dynamics", J. Differ. Equations, 25, 342-364, (1977). 1.2

[15] Batt, J., Faltenbacher, W., and Horst, E., "Stationary spherically symmetric models in stellar dynamics", Arch. Ration. Mech. Anal., 93, 159-183, (1986). 3

[16] Berger, B.K., Chruściel, P.T., Isenberg, J.A., and Moncrief, V., "Global foliations of vacuum spacetimes with $T^{2}$ isometry", Ann. Phys. (N.Y.), 260, 117-148, (1997). 2.2

Living Reviews in Relativity

http://www. livingreviews.org/lrr-2005-2 
[17] Bouchut, F., Golse, F., and Pallard, C., "Classical solutions and the Glassey-Strauss theorem for the 3D Vlasov-Maxwell system", Arch. Ration. Mech. Anal., 170, 1-15, (2003). 1.2

[18] Burnett, G.A., and Rendall, A.D., "Existence of maximal hypersurfaces in some spherically symmetric spacetimes", Class. Quantum Grav., 13, 111-123, (1997). 2.2

[19] Calogero, S., "Spherically symmetric steady states of galactic dynamics in scalar gravity", Class. Quantum Grav., 20, 1729-1741, (2003). 1.3

[20] Calogero, S., "The Newtonian limit of the relativistic Boltzmann equation", J. Math. Phys., 45, 4042-4052, (2004). 1.1

[21] Calogero, S., and Lee, H., "The non-relativistic limit of the Nordström-Vlasov system", Commun. Math. Sci., 2, 19-34, (2004). 1.3

[22] Calogero, S., and Rein, G., "On classical solutions of the Nordström-Vlasov system", Commun. Part. Diff. Eq., 28, 1863-1885, (2003). 1.3

[23] Calogero, S., and Rein, G., "Global weak solutions to the Nordström-Vlasov system", $J$. Differ. Equations, 204, 323-338, (2004). 1.3

[24] Cercignani, C., Illner, R., and Pulvirenti, M., The mathematical theory of dilute gases, vol. 106 of Applied Mathematical Sciences, (Springer, New York, U.S.A., 1994). 1.1

[25] Choquet-Bruhat, Y., "Problème de Cauchy pour le système intégro différentiel d'EinsteinLiouville", Ann. Inst. Fourier, 21, 181-201, (1971). 1.4

[26] Choquet-Bruhat, Y., and Noutchegueme, N., "Systéme de Yang-Mills-Vlasov en jauge temporelle", Ann. Inst. Henri Poincare, 55, 759-787, (1991). 1.4

[27] Christodoulou, D., "The problem of a self-gravitating scalar field", Commun. Math. Phys., 105, 337-361, (1986). 2.1

[28] Christodoulou, D., "The formation of black holes and singularities in spherically symmetric gravitational collapse", Commun. Pure Appl. Math., 44, 339-373, (1991). 2.1

[29] Christodoulou, D., "Examples of naked singularity formation in the gravitational collapse of a scalar field", Ann. Math., 140, 607-653, (1994). 2.1

[30] Christodoulou, D., "Self-gravitating relativistic fluids: the formation of a free phase boundary in the phase transition from soft to hard", Arch. Ration. Mech. Anal., 134, 97-154, (1996). 1.4

[31] Christodoulou, D., "The instability of naked singularities in the gravitational collapse of a scalar field", Ann. Math., 149, 183-217, (1999). 2.1

[32] Dafermos, M., "Spherically symmetric spacetimes with a trapped surface", (2004). URL (cited on 22 February 2005):

http://arXiv.org/abs/gr-qc/0403032. 2.1

[33] Dafermos, M., and Rendall, A.D., "An extensionprinciple for the Einstein-Vlasov system in spherical symmetry", (2004). URL (cited on 22 February 2005):

http://arXiv.org/abs/gr-qc/0411075. 2.1

[34] de Groot, S.R., van Leeuwen, W.A., and van Weert, C.G., Relativistic Kinetic Theory: Principles and Applications, (North-Holland, Amsterdam, Netherlands; New York, U.S.A., 1980). 1.1, 1.1 
[35] DiPerna, R.J., and Lions, P.-L., "Global weak solutions of Vlasov-Maxwell systems", Commun. Pure Appl. Math., 42, 729-757, (1989). 1.2, 1.3

[36] DiPerna, R.J., and Lions, P.-L., "On the Cauchy problem for Boltzmann equations: global existence and weak stability", Ann. Math., 130, 321-366, (1989). 1.1

[37] Dudyński, M., and Ekiel-Jezewska, M.L., "Global existence proof for relativistic Boltzmann equation", J. Stat. Phys., 66, 991-1001, (1992). 1.1

[38] Ehlers, J., "Survey of general relativity theory", in Israel, W., ed., Relativity, Astrophysics and Cosmology, Proceedings of the summer school held 14-26 August 1972 at the Banff Centre, Banff, Alberta, vol. 38 of Atrophysics and Space Science Library, (Reidel, Dordrecht, Netherlands; Boston, U.S.A., 1973). 1.4

[39] Fjällborg, M., "On the cylindrically symmetric Einstein-Vlasov system", unknown status. Preprint Karlstad University Studies 2004:19. 2

[40] Ganguly, K., and Victory, H., "On the convergence for particle methods for multidimensional Vlasov-Poisson systems", SIAM J. Numer. Anal., 26, 249-288, (1989). 2.1

[41] Glassey, R.T., The Cauchy Problem in Kinetic Theory, (SIAM, Philadelphia, U.S.A., 1996). $1.1,1.2$

[42] Glassey, R.T., and Schaeffer, J., "On symmetric solutions to the relativistic Vlasov-Poisson system", Commun. Math. Phys., 101, 459-473, (1985).1.2, 1.2

[43] Glassey, R.T., and Schaeffer, J., "The "two and one-half dimensional" relativistic VlasovMaxwell system", Commun. Math. Phys., 185, 257-284, (1997). 1.2

[44] Glassey, R.T., and Schaeffer, J., "The relativistic Vlasov Maxwell system in two space dimensions: part II", Arch. Ration. Mech. Anal., 141, 355-374, (1998). 1.2, 1.3

[45] Glassey, R.T., and Schaeffer, J., "On global symmetric solutions to the relativistic VlasovPoisson equation in three space dimensions", Math. Method. Appl. Sci., 24, 143-157, (2001). 1.2

[46] Glassey, R.T., and Strauss, W., "Singularity formation in a collisionless plasma could only occur at high velocities", Arch. Ration. Mech. Anal., 92, 56-90, (1986). 1.2, 1.3, 2.1

[47] Glassey, R.T., and Strauss, W., "Absence of shocks in an initially dilute collisionless plasma", Commun. Math. Phys., 113, 191-208, (1987). 1.2

[48] Glassey, R.T., and Strauss, W., "Asymptotic stability of the relativistic Maxwellian", Publ. Res. Inst. Math. Sci., 29, 301-347, (1992). 1.1, 1.1

[49] Glassey, R.T., and Strauss, W., "Asymptotic stability of the relativistic Maxwellian", Transp. Theor. Stat. Phys., 24, 657-678, (1995). 1.1

[50] Gundlach, C., "Critical phenomena in gravitational collapse", Adv. Theor. Math. Phys., 2, $1-49,(1998) . \quad 2.1$

[51] Guo, Y., and Rein, G., "Isotropic steady states in stellar dynamics", Commun. Math. Phys., 219, 607-629, (2001). 3

[52] Henkel, O., "Global prescribed mean curvature foliations in cosmological spacetimes with matter, Part I", J. Math. Phys., 43, 2439-2465, (2002). 2.2 
[53] Henkel, O., "Global prescribed mean curvature foliations in cosmological spacetimes with matter, Part II", J. Math. Phys., 43, 2466-2485, (2002). 2.2

[54] Horst, E., "On the classical solutions of the initial value problem for the unmodified nonlinear Vlasov equation (Parts I and II)", Math. Method. Appl. Sci., 6, 262-279, (1982). 1.2

[55] Horst, E., "On the asymptotic growth of the solutions of the Vlasov-Poisson system", Math. Method. Appl. Sci., 16, 75-86, (1993). 1.2

[56] Horst, E., and Hunze, R., "Weak solutions of the initial value problem for the unmodified nonlinear Vlasov equation", Math. Method. Appl. Sci., 6, 262-279, (1984). 1.2

[57] Illner, R., and Rein, G., "Time decay of the solutions of the Vlasov-Poisson system in the plasma physical case", Math. Method. Appl. Sci., 19, 1409-1413, (1996). 1.2

[58] Illner, R., and Shinbrot, M., "The Boltzmann equation, global existence for a rare gas in an infinite vacuum", Commun. Math. Phys., 95, 217-226, (1984). 1.1

[59] Isenberg, J.A., and Rendall, A.D., "Cosmological spacetimes not covered by a constant mean curvature slicing", Class. Quantum Grav., 15, 3679-3688, (1998). 2.2

[60] Klainerman, S., and Staffilani, G., "A new approach to study the Vlasov-Maxwell system", Commun. Pure Appl. Anal., 1, 103-125, (2002). 1.2

[61] Kunze, M., and Rendall, A.D., "The Vlasov-Poisson system with radiation damping", Ann. Inst. Henri Poincare, 2, 857-886, (2001). 1.2

[62] Lee, H., "Global existence of solutions of the Nordström-Vlasov system in two space dimensions", (2003). URL (cited on 22 February 2005):

http://arXiv.org/abs/math-ph/0312014. 1.3

[63] Lee, H., "Asymptotic behaviour of the Einstein-Vlasov system with a positive cosmological constant", Math. Proc. Camb. Phil. Soc., 137, 495-509, (2004). 2.3

[64] Lee, H., "The Einstein-Vlasov system with a scalar field", (2004). URL (cited on 22 February 2005):

http://arXiv.org/abs/gr-qc/0404007. 2.3

[65] Lions, P.-L., "Compactness in Boltzmann's equation via Fourier integral operators and applications. I.", J. Math. Kyoto Univ., 34, 391-427, (1994). 1.1

[66] Lions, P.-L., and Perthame, B., "Propagation of moments and regularity for the 3dimensional Vlasov-Poisson system", Invent. Math., 105, 415-430, (1991). 1.2

[67] Makino, T., "On spherically symmetric stellar models in general relativity", J. Math. Kyoto Univ., 38, 55-69, (1998). 3

[68] Martín-García, J.M., and Gundlach, C., "Self-similar spherically symmetric solutions of the massless Einstein-Vlasov system", Phys. Rev. D, 65, 084026, (2002). 2.1

[69] Nishida, T., and Imai, K., "Global solutions to the initial value problem for the nonlinear Boltzmann equation", Publ. Res. Inst. Math. Sci., 12, 229-239, (1976). 1.1

[70] Nordström, G., "Zur Theorie der Gravitation vom Standpunkt des Relativitätsprinzips", Ann. Phys. (Leipzig), 42, 533, (1913). 1.3 
[71] Noundjeu, P., "On a regularity theorem for solutions of the spherically symmetric EinsteinVlasov-Maxwell system", (2004). URL (cited on 22 February 2005):

http://arXiv.org/abs/gr-qc/0406021. 2.1

[72] Noundjeu, P., and Noutchegueme, N., "Local Existence and Continuation Criterion for Solutions of the Spherically Symmetric Einstein-Vlasov-Maxwell System", Gen. Relativ. Gravit., 36, 1572-9532, (2004). Related online version (cited on 22 February 2005): http://arXiv.org/abs/gr-qc/0311081. 2.1

[73] Noundjeu, P., Noutchegueme, N., and Rendall, A.D., "Existence of initial data satisfying the constraints for the spherically symmetric Einstein-Vlasov-Maxwell system", J. Math. Phys., 45, 668-676, (2004). 2.1

[74] Noutchegueme, N., and Tetsadjio, M.E., "Global solutions for the relativistic Boltzmann equation in the homogeneous case on the Minkowski space-time", (2003). URL (cited on 22 February 2005):

http://arXiv.org/abs/gr-qc/0307065. 1.1

[75] Olabarrieta, I., and Choptuik, M.W., "Critical phenomena at the threshold of black hole formation for collisionless matter in spherical symmetry", Phys. Rev. D, 65, 024007, (2002). 2.1

[76] Pallard, C., "On global smooth solutions to the 3D Vlasov-Nordström system", submitted. To appear in Annales de l'I.H.P. Analyse non linéaire. 1.3

[77] Pallard, C., "On the boundedness of the momentum support of solutions to the relativistic Vlasov-Maxwell system", submitted. To appear in Indiana Univ. Math. J. 1.2

[78] Pallard, C., "A pointwise bound on the electromagnetic field generated by a collisionless plasma", unknown status. Preprint. 1.2

[79] Perthame, B., "Time decay, propagation of low moments and dispersive effects for kinetic equations", Commun. Part. Diff. Eq., 21, 659-686, (1996). 1.2

[80] Pfaffelmoser, K., "Global classical solutions of the Vlasov-Poisson system in three dimensions for general initial data", J. Differ. Equations, 95, 281-303, (1992). 1.2

[81] Rein, G., "Static solutions of the spherically symmetric Vlasov-Einstein system", Math. Proc. Camb. Phil. Soc., 115, 559-570, (1994). 3

[82] Rein, G., "Cosmological solutions of the Vlasov-Einstein system with spherical, plane, and hyperbolic symmetry", Math. Proc. Camb. Phil. Soc., 119, 739-762, (1996). 2.2, 2.2, 2.3

[83] Rein, G., "Growth estimates for the Vlasov-Poisson system in the plasma physics case", Math. Nachr., 191, 269-278, (1998). 1.2

[84] Rein, G., "Static shells for the Vlasov-Poisson and Vlasov-Einstein systems", Indiana Univ. Math. J., 48, 335-346, (1999). 3

[85] Rein, G., "Stationary and static stellar dynamical models with axial symmetry", Nonlinear Anal., 41, 313-344, (2000). 3

[86] Rein, G., "Global weak solutions of the relativistic Vlasov-Maxwell system revisited", Commun. Math. Sci., 2, 145-148, (2004). 1.2 
[87] Rein, G., "On future completeness for the Einstein-Vlasov system with hyperbolic symmtery", Math. Proc. Camb. Phil. Soc., 137, 237-244, (2004). 2.2, 2.3

[88] Rein, G., and Rendall, A.D., "Global existence of solutions of the spherically symmetric Vlasov-Einstein system with small initial data", Commun. Math. Phys., 150, 561-583, (1992). Erratum: Commun. Math. Phys. 176, (1996), 475-478. 2.1, 2.1

[89] Rein, G., and Rendall, A.D., "The Newtonian limit of the spherically symmetric VlasovEinstein system", Commun. Math. Phys., 150, 585-591, (1992). 2.1, 3

[90] Rein, G., and Rendall, A.D., "Smooth static solutions of the spherically symmetric VlasovEinstein system", Ann. Inst. Henri Poincare A, 59, 383-397, (1993). 3

[91] Rein, G., and Rendall, A.D., "Compact support of spherically symmetric equilibria in relativistic and non-relativistic galactic dynamics", Math. Proc. Camb. Phil. Soc., 128, 363-380, (2000). 3

[92] Rein, G., Rendall, A.D., and Schaeffer, J., "A regularity theorem for solutions of the spherically symmetric Vlasov-Einstein system", Commun. Math. Phys., 168, 467-478, (1995). 2.1

[93] Rein, G., Rendall, A.D., and Schaeffer, J., "Critical collapse of collisionless matter- a numerical investigation", Phys. Rev. D, 58, 044007, (1998). 2.1

[94] Rein, G., and Rodewis, T., "Convergence of a particle-in-cell scheme for the spherically symmetric Vlasov-Einstein system", Indiana Univ. Math. J., 52, 821-862, (2003). 2.1

[95] Rendall, A.D., "Cosmic censorship and the Vlasov equation", Class. Quantum Grav., 9, L99-L104, (1992). 2.1

[96] Rendall, A.D., "The Newtonian limit for asymptotically flat solutions of the Einstein-Vlasov system", Commun. Math. Phys., 163, 89-112, (1994). 2.1

[97] Rendall, A.D., "Crushing singularities in spacetimes with spherical, plane and hyperbolic symmetry", Class. Quantum Grav., 12, 1517-1533, (1995). 2.2

[98] Rendall, A.D., "Global properties of locally spatially homogeneous cosmological models with matter", Math. Proc. Camb. Phil. Soc., 118, 511-526, (1995). 2.2

[99] Rendall, A.D., "Existence and non-existence results for global constant mean curvature foliations", Nonlinear Anal., 30, 3589-3598, (1997). 2.2

[100] Rendall, A.D., "Existence of constant mean curvature foliations in spacetimes with twodimensional local symmetry", Commun. Math. Phys., 189, 145-164, (1997). 2.2, 2.2

[101] Rendall, A.D., "An introduction to the Einstein-Vlasov system", in Chruściel, P.T., ed., Mathematics of Gravitation, Part I: Lorentzian Geometry and Einstein Equations, Proceedings of the Workshop Mathematical Aspects of Theories of Gravitation, held in the Banach Center of the Institute of Mathematics of the Polish Academy of Sciences, Warsaw, Poland, February 29 - March 30, 1996, vol. 41(I) of Banach Center Publications, 35-68, (Polish Academy of Sciences, Institute of Mathematics, Warsaw, Poland, 1997). 1.4, 2, 2.1

[102] Rendall, A.D., "Cosmological models and centre manifold theory", Gen. Relativ. Gravit., 34, 1277-1294, (2002). Related online version (cited on 22 February 2005):

http://arXiv.org/abs/gr-qc/0112040. 2.2 
[103] Rendall, A.D., and Tod, K.P., "Dynamics of spatially homogeneous solutions of the EinsteinVlasov equations which are locally rotationally symmetric", Class. Quantum Grav., 16, 17051726, (1999). 2.2

[104] Rendall, A.D., and Uggla, C., "Dynamics of spatially homogeneous locally rotationally symmetric solutions of the Einstein-Vlasov equations", Class. Quantum Grav., 17, 4697-4714, (2000). 2.2

[105] Schaeffer, J., "The classical limit of the relativistic Vlasov-Maxwell system", Commun. Math. Phys., 104, 403-421, (1986). 1.2

[106] Schaeffer, J., "Discrete approximation of the Poisson-Vlasov system", Quart. Appl. Math., 45, 59-73, (1987). 2.1

[107] Schaeffer, J., "A class of counterexamples to Jeans' theorem for the Einstein-Vlasov system", Commun. Math. Phys., 204, 313-327, (1999). 1.2, 3

[108] Shizuta, Y., "On the classical solutions of the Boltzmann equation", Commun. Pure Appl. Math., 36, 705-754, (1983). 1.1

[109] Stewart, J.M., Non-equilibrium relativistic kinetic theory, vol. 10 of Lecture Notes in Physics, (Springer, Berlin, Germany; New York, U.S.A., 1971). 1.4

[110] Synge, J.L., The relativistic gas, (North-Holland; Interscience, Amsterdam, Netherlands; New York, U.S.A., 1957). 1.1

[111] Tchapnda, S.B., "Structure of solutions near the initial singularity for the surface-symmetric Einstein-Vlasov system", Class. Quantum Grav., 21, 5333-5346, (2004). Related online version (cited on 22 February 2005): http://arXiv.org/abs/gr-qc/0407062. 2.3, 2.3

[112] Tchapnda, S.B., and Noutchegueme, N., "The surface-symmetric Einstein-Vlasov system with cosmological constant", (2003). URL (cited on 22 February 2005):

http://arXiv.org/abs/gr-qc/0304098. 2.3

[113] Tchapnda, S.B., and Rendall, A.D., "Global existence and asymptotic behaviour in the future for the Einstein-Vlasov system with positive cosmological constant", Class. Quantum Grav., 20, 3037-3049, (2003). 2.3

[114] Tegankong, D., Noutchegueme, N., and Rendall, A.D., "Local existence and continuation criteria for solutions of the Einstein-Vlasov-scalar field system with surface symmetry", $J$. Hyperbol. Differ. Equations, 1, 691-724, (2004). Related online version (cited on 22 February 2005):

http://arXiv.org/abs/gr-qc/0405039. 2.3

[115] Ukai, S., "On the existence of global solutions of a mixed problem for the nonlinear Boltzmann equation", Proc. Japan Acad., 50, 179-184, (1974). 1.1

[116] Villani, C., "A review of mathematical topics in collisional kinetic theory", in Friedlander, S., and Serre, D., eds., Handbook of Mathematical Fluid Dynamics, Vol. 1, 71-305, (Elsevier, Amsterdam, Netherlands; Boston, U.S.A., 2002). Related online version (cited on 22 February 2005):

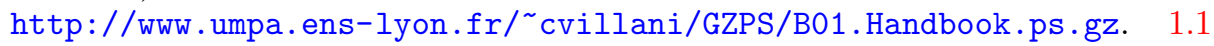

[117] Wald, R.M., General Relativity, (University of Chicago Press, Chicago, U.S.A., 1984). 1.4

Living Reviews in Relativity

http: //www . livingreviews . org/lrr-2005-2 
[118] Weaver, M., "On the area of the symmetry orbits in $T^{2}$ symmetric spacetimes with Vlasov matter", Class. Quantum Grav., 21, 1079-1098, (2004). 2.2, 2.2, 2.3

[119] Wennberg, B., "Regularity in the Boltzmann equation and the Radon transform", Commun. Part. Diff. Eq., 19, 2057-2074, (1994). 1.1

[120] Wennberg, B., "The geometry of binary collisions and generalized Radon transforms", Arch. Ration. Mech. Anal., 139, 291-302, (1997). 1.1

[121] Wolansky, G., "Static solutions of the Einstein-Vlasov system", Arch. Ration. Mech. Anal., 156, 205-230, (2001). $\quad 3$ 